\title{
FREQUENCY OF METABOLIC SYNDROME AMONG STROKE PATIENTS
}

\author{
AHMEDUL KABIR $^{1}$, MM SADEKA $^{2}$, M J AHMED ${ }^{3}$, E KABIR $^{4}$, MA KAHHAR $^{5}$
}

\begin{abstract}
:
Background: Metabolic syndrome is a cluster of cardiovascular risk factor and is strongly associated with CVD. This study had been carried out at Mitford Hospital with a view to get a profile of the stroke patients and to obtain the proportion of the patients having metabolic syndrome.

Patients \& methods: In the observational study a total of fifty patients of stroke (confirmed by CT Scan) were studied at medical unit of Mitford hospital from December, 2005 to April, 2006.

Results: Among the fifty stroke patients, about half (48\%) were diagnosed as metabolic syndrome and there was slight female preponderance among them. Majority of the patients (79\%) were from middle socio-economic class. Investigations regarding hypertension, diabetes mellitus and dyslipidaemia revealed the percentages of these risk factors along with gender distributions among the patients with stroke.

Conclusion: Stage two hypertension and diabetes were the major risk factors causing stroke. This study would provide baseline line information for the future researcher in the relevant field.
\end{abstract}

\section{Introduction:}

The metabolic syndrome, has long been recognized as a cluster of coronary artery disease risk factor. In 1988, Reaven and colleagues, described the syndrome as a link between insulin resistance and hypertension, dyslipidemia, type 2 diabetes and other metabolic abnormalities that carries greater risk for cardiovascular disease. ${ }^{1}$ The ATPIII (third report of the National Cholesterol Education Program Adult Treatment Panel) clinical definition of the metabolic syndrome $^{2}$ requires the presence of three or more of the following: (1) abdominal obesity (waist circumference $\geq 102 \mathrm{~cm}$ in men and $\geq 88 \mathrm{~cm}$ in women); (2) a high triglyceride level ( $>150 \mathrm{mg} / \mathrm{dl}$ or $>1.69$ $\mathrm{mmol} / \mathrm{L})$; (3) a low HDL cholesterol level $(<40 \mathrm{mg} / \mathrm{dl}$ or $<1.03 \mathrm{mmol} / \mathrm{L}$ for men and $<50 \mathrm{mg} / \mathrm{dl}$ or $<1.29$ $\mathrm{mmol} / \mathrm{L}$ for women); (4) high blood pressure (systolic $\geq 130 \mathrm{~mm} \mathrm{Hg}$ or diastolic $\geq 85 \mathrm{~mm} \mathrm{Hg}$ ) and (5) a high fasting plasma glucose concentration $(\geq 110 \mathrm{mg} / \mathrm{dl})$.

The mechanisms underlying the metabolic syndrome are not fully known; however, insulin resistance seems to modify biochemical responses in a way that predisposes to metabolic risk factors. Obesity has been strongly associated with insulin resistance and it is not the BMI rather central adiposity is more responsible for development of the syndrome. ${ }^{3}$ The Insulin resistance is also related to essential hypertension and insulin levels have been found to be significantly higher in patients with essential hypertension. ${ }^{4}$ Several mechanisms have been hypothesized whereby insulin resistance play a major role in dyslipidemia. Hyperinsulinemia is known to enhance hepatic very low density lipoprotein synthesis and thus may directly contribute to the increased plasma trigyceride and LDL cholesterol level. ${ }^{5}$ Resistance to the action of insulin on lipoprotein lipase in peripheral tissues may also contribute to elevated triglyceride and LDL cholesterol levels. ${ }^{6}$

Obesity is an important modulator of metabolic syndrome and it has been postulated that childhood obesity increases the risk of metabolic syndrome in adulthood. ${ }^{7}$ The prevalence of metabolic syndrome is

1. Resident Physician, Department of Medicine, Dhaka Medical College Hospital, Dhaka

2. Indoor Medical Officer, Sir Salimullah Medical College \& Mitford Hospital, Dhaka

3. Honorary Medical Officer, Sir Salimullah Medical College \& Mitford Hospital, Dhaka

4. Asst. Registrar, Sir Salimullah Medical College \& Mitford Hospital, Dhaka

5. Professor, Department of Medicine, Sir Salimullah Medical College \& Mitford Hospital, Dhaka 
increasing day by day as is the degree of obesity is increasing among all classes of people. In the screening phase of NAVIGATOR trial the prevalence of metabolic syndrome was calculated to be $51 \% .{ }^{7}$ When divided by region (North America, Latin America, Europe and Asia) rates of the metabolic syndrome were lowest in Asians $(36 \%)^{7}$ thereby it has been suggested that the current criteria of metabolic syndrome may need to be modified among the Asians. ${ }^{8}$ This study also confirmed the need to identify the patients with metabolic syndrome in order to prevent the development of diabetes and cardiovascular disease.

Since the metabolic syndrome is highly correlated with CVD, an effort had been made to find out the presence of the syndrome among the stroke patients. With this objective fifty patients with stroke were studied at medicine unit in Sir Salimullah Medical College and Mitford hospital from December 2005 to April 2006. This paper will shortly discuss the profile of those patients.

\section{Materials and method:}

The admitted patients with sudden onset of characteristic neurological deficit and having distinctive neurological signs reflecting the regions of brain involved were recruited for the study. Patients with loss of consciousness due to head injury, metabolic coma, trauma, poisoning or epilepsy were not included. Elaborated history regarding the illness was obtained from each patient's attendant with specific attention to hypertention and diabetes mellitus. Complete clinical examination was performed with a wide range of clinical examination including fasting and postprandial blood glucose, urine RME, ECG, serum creatinine, chest X-ray, fasting lipid profile and CT scan of the brain. All these information were compiled in a data sheet and checked and edited. From the data some descriptive statistics and graphical picture were obtained.

\section{Case definition:}

Metabolic syndrome was diagnosed as per ATPIII criteria.

\section{Results:}

A total of fifty pateitnts, 20 male and 30 female were admitted with inclusion criteria. Among the fifty stroke patients, 24 (48\%) were diagnosed with metabolic syndrome. Among them 11(45.83\%) was male and the rest, that is $13(54.16 \%)$ were female (fig: 1 ).

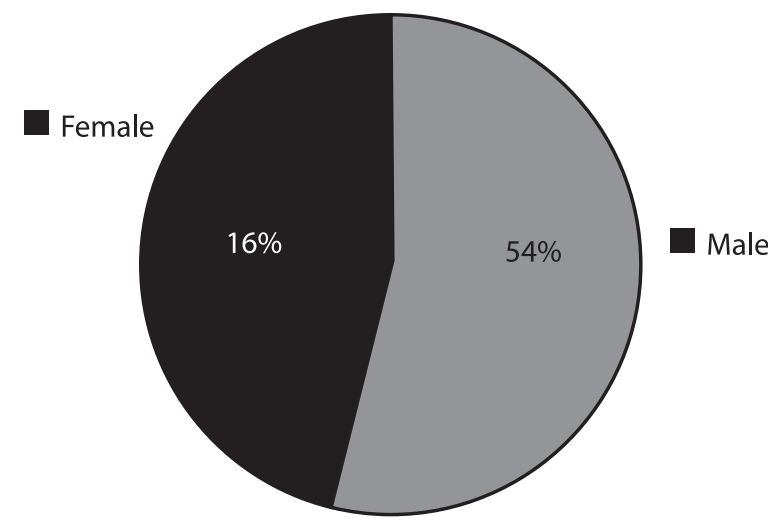

Fig.-1: Distribution of gender among the patients

The majority of the patients (19/79.16\%) with metabolic syndrome came to the hospital belonged to middle class family(fig:2). Among these patients $52.43 \%$ was female and the rest $47.36 \%$ was male.

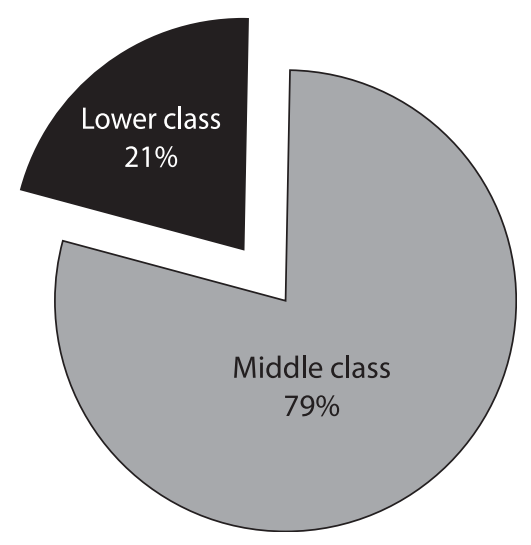

Fig.-2: Distribution of the social class among the patients

Investigations of the patient revealed the stage of hypertension, diabetic status and lipid profile of the patients. These information was compiled in bar diagram(fig: 3 ) and showed that $37.5 \%$ patients had stage 1 hypertension and $62.5 \%$ had stage 2 hypertension. Fifty eight percent patients was diagnosed as diabetic , 29.16\% had impaired glucose tolerance and $12.5 \%$ patient had impaired fasting glucose. Fasting lipid profile of the patients revealed that $41.66 \%$ had increased triglyceride level and $33.33 \%$ had increased LDL cholesterol level. Combined dyslipidemia was found in $25 \%$ pastients.

CT scan of the brain demonstrated infarction or hemorrhage as the cause of stroke. Fifteen (62.50\%) patients were found to have infarctive stroke and 9(37.50\%) patients had haemorrhagic stroke. 


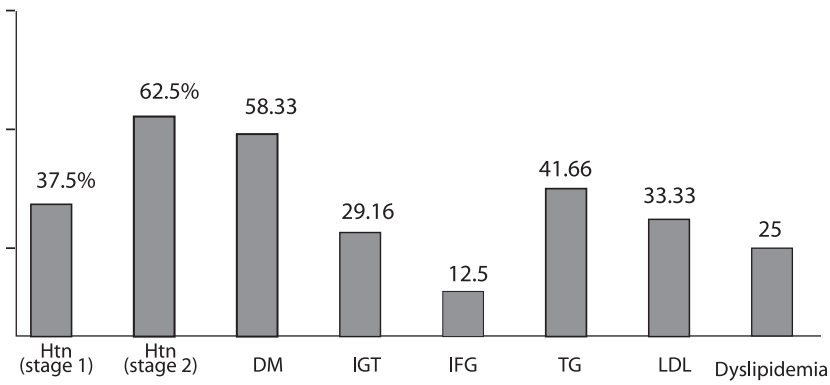

Fig.-3: Percentage of the patients having hypertension (stage one and two), Diabetes Mellitus (DM), Impaired glucose tolerance test (IGT), Impaired fasting glucose, triglyceridemia, Increased LDL level and combined dyslipidemia

Figure 4 is a bar diagram showing the gender distributions of the cardiovascular risk factors. In case of stage one hypertension male(44.44\%) and female (55.55\%) are almost equally affected. In case of stage two hypertension, female (66.66\%) is suffering almost twice than that of the male(33.33\%). In the study, diabetes were found to be present among $64.28 \%$ female and $35.71 \%$ male. Impaired glucose tolerance and impaired fasting glucose were shown different distribution among different sexes. In case of impaired glucose tolerance, the male to female ratio was (28.37:71.42), whereas in case of impaired fasting glucose the ratio was (66.66:33.33). Seventy percent male had hypertriglyceridemia, whereas only $30 \%$ female had high triglyceride level. LDL level showed almost similar male female distribution (62.5: 37.5). Thus combined dyslipidemia was more among the male that is $83.33 \%$.

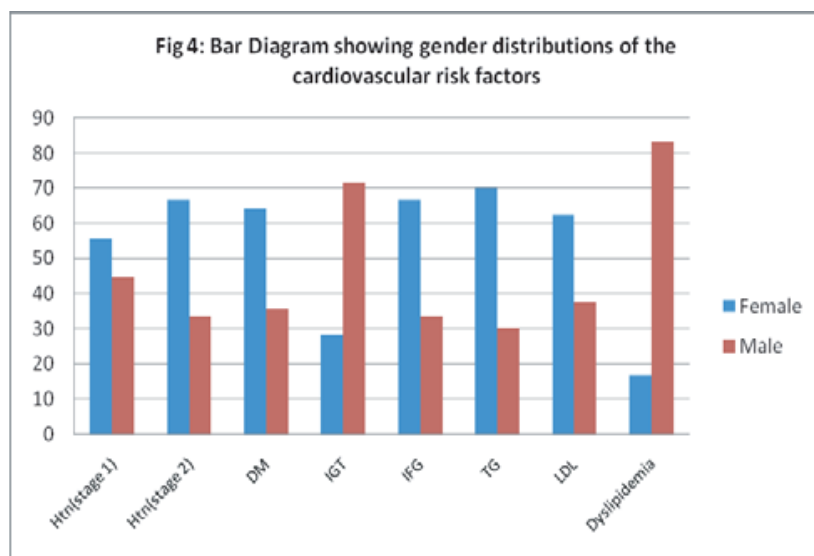

Fig.-4: Diagram showing gender distributions of the cardiovascular risk factors

\section{Discussion:}

This study was carried out in order to get a profile of the stroke patients admitted at Mitford Hospital in terms of cardiovascular risk factors. About half of the admitted patients were diagnosed with metabolic syndrome. The prevalence of metabolic syndrome in the adult population in developed countries is $22-39 \%$.

There was slight female preponderance among the patients having metabolic syndrome. Possible explanations for these differences include disparities in the prevalence and potency of vascular risk factors. In Framingham, women with 3 or more metabolically linked factors had a relative risk of 5.9 (95\% CI, 2.5 to 13.7) for coronary artery disease compared with a relative risk of 2.3 (95\% CI, 1.6 to 2.4) in men. Data from the National Health and Nutrition Examination Survey identified more vascular risk factors among women than men. Alternative explanations for possible sex differences include a greater impact of the metabolic syndrome among postmenopausal women. Our data on menopause and hormone use in our study are incomplete. Clearly this question warrants further investigation. ${ }^{9-11}$

Majority belonged to middle social class who were less likely to have completed high school. Although these classification of social resource access were adjusted for our models, there may be a number of other social resources, both at the individual level and population level, which need further exploration.

Regarding the cause of stroke, as revealed by CT scan, majority had stroke due to infarction. In case of cardiovascular risk factors, descriptive statistics showed the proportions of cardiovascular risk factors among the patients and their gender distributions was also shown in the study. Stage two hypertension and diabetes were most evident among the patients. Therefore, it could possibly be inferred that, hypertension has been proved to be the silent killer once again. Regarding the gender distribution, overall the female patients were shown to be more sufferer of dyslipidemia and impaired glucose tolerance.

The increased risk of cardiovascular disease in patients with the metabolic syndrome is probably associated with the inflammatory and hypercoagulable states that may occur in these patients. In the presence of visceral obesity, several circulating inflammatory adipokines, including tumor necrosis factor (TNF), leptin, interleukin-6 (IL-6) and angiotensinogen, are produced by the adipose tissue, thus underlying the pivotal role 
of visceral obesity in this syndrome. TNF inhibits insulin signaling thus contributing to insulin resistance. Leptin activates the immune system and increases blood pressure. IL- 6 stimulates the hepatic production of C-reactive protein in obese subjects. Angiotensin II (produced from angiotensinogen) exerts its adverse endocrine effects via the angiotensin II type 1 receptor (AT1), leading to oxidative stress, vasoconstriction, aldosterone secretion, renal sodium resorption, sympathetic stimulation, vasopressin release, plasminogen activator inhibitor-1 (PAI-1) expression and, possibly, to thrombosis. In contrast to these adipokines, adiponectin, a circulating collagenlike molecule also produced by adipose tissue, is inversely correlated with the fat mass in obese subjects. Adiponectin enhances insulin sensitivity and inhibits inflammatory effects on the endothelium, largely through inhibiting TNF activation of nuclear factor B. Furthermore, adiponectin stimulates the production of nitric oxide in endothelial cells, and serum levels of C-reactive protein are reciprocally associated with adiponectin levels, thus suggesting a potential link between adiponectin and coronary artery disease. $^{12}$

The metabolic syndrome is also associated with increased plasma levels of fibrinogen, factor VII and factor VIII, thus leading to a potential hypercoagulable state, and with increased levels of PAI-1, thus leading to a hypofibrinolytic state. Increased levels of fibrinogen are associated with both chronic inflammation and insulin resistance in the metabolic syndrome. Low-grade chronic inflammation has been associated with increased release of soluble tissue factor and factor VII. Furthermore, factor VII activity correlates with both body mass index and triglyceride levels. The simultaneous increase in both soluble tissue factor and factor VII clearly enhances the risk of activation of the coagulation cascade. Increased levels PAI- 1 are common in subjects with the metabolic syndrome. Insulin resistance and chronic inflammation contribute to the increase in PAI-1. Finally, decreased plasma tissue plasminogen activator activity is related to insulin resistance in patients with characteristics of the metabolic syndrome. ${ }^{12}$

Overall, these changes contribute to attenuation of plasminogen conversion, resulting in a hypofibrinolytic state. Finally, endothelial dysfunction (measured by flow-mediated dilation to assess nitric oxide bioavailability) is commonly found in patients with the metabolic syndrome, and is directly correlated with increased cardiovascular risk. The induction of endothelial dysfunction has been associated with the presence of hyperinsulinemia and dyslipidemia. Platelets from obese insulin-resistant subjects have reduced sensitivity to the anti-aggregatory effects of insulin. Moreover, very-low-density lipoprotein and triglycerides have been shown to increase platelet aggregability, whereas this effect is reversed by HDL cholesterol. ${ }^{12}$

\section{Conclusion:}

The metabolic syndrome has been shown to be an important cluster of cardiovascular risk factors placing patients at an increased risk of coronary artery disease and ischemic stroke. Thus, diagnosing and adequately managing the metabolic syndrome is an important step in the process of preventing cardiovascular disease. Greater emphasis needs to be placed on the early diagnosis and treatment of patients at risk for vascular disease. Further understanding of gender and social class differences in terms of their impact on the metabolic syndrome will help us effectively target populations at increased risk of ischemic stroke.

This study, has given the baseline information about the pattern of stroke patients in a tertiary care hospital. This data need to be evaluated further, with extension of the relevant information. Therefore, further study is mandatory to obtain information to formulate any health policy. However, this small scale study would serve as a preliminary guideline for the future researcher.

\section{References:}

1. Weiss R, Dziura J, Burgert TS et al. Obesity and Metabolic Syndrome in Children and Adolescents. N Eng J Med 2004; 350: 2362-74.

2. Park Y, Zhu S, Palaniappan L et al. The Metabolic Syndrome: Prevalence and Associated Risk Factor Findings in the US Population From the Third National Health and Nutrition Examination Survey, 1988-1994. Arch Intern Med 2003;163:427-436.

3. Steinberger J, Daniel SR. Obesity, Insulin Resistance, Diabetes and Cardiovascular Risk in Children. An American Heart Association Scientific Statement From the Atherosclerosis, Hypertension and Obesity in the Young Committee (Ciouncil in Cardiovascular Disease in the Young) and the Diabetes Committee (Council on Nutrition, Physical Activity and Metabolism). 
4. Ferrannini E, Haffner SM, Stern MP. Essential Hypertension: An Insulin Resistant State. J Cardiovasc Pharmacol 1990; 15: 18-25.

5. Stalder M, Pometta D, Suenram A. Relationship between plasma insulin levels and high density lipoprotein cholesterol level in healthy men. Diabetologia 1981; 21: 544-548.

6. Sadur CN, Yost TJ, Eckel RH. Insulin responsiveness of adipose tissue lipoprotein lipase is delayed but preserved in obesity. J clin Endocrinol Metab 1984; 59: 1176-1182.

7. Vanhala M, Vanhala P, Kumpusalu E et al. Relation between obesity from childhood to adulthood and the metabolic syndrome: population based study. BMJ 1998; 317: 319.

8. Peters AL. The broadening domain of Metabolic syndrome.
9. Holman R, Haffner S, Macmurrey JJ et al. Regional differences in proportion of subjects with cardiovascular disease screened for the NAVIGATOR trial who have the metabolic syndrome. Programs and abstracts of the $65^{\text {th }}$ scientific sessions of the American Diabetes Association; June10-14, 2005; San Diego, California. Abstract 1062-p.

10. Khunti K, Davies M. Metabolic Syndrome. BMJ 2005; 331: 1153-1154.

11. Boden A, Bernadette, Sacco, Ralph L et al. Metabolic Syndrome and Ischemic Stroke Risk: Northern Manhattan Study, Stroke. American Heart Association 2008; 39: 30-35.

12. Francesco D, Erica R, Walter A. The metabolic syndrome and the risk of thrombosis. Haematologica 92: 297-299. 